\title{
Cirugía radioguíada con análogos de somatostatina radiomarcados en tumores neuroendocrinos: reporte de caso
}

\section{Radioguided surgery with radiolabeled somatostatin analogues in neuroendocrine tumors: Case report}

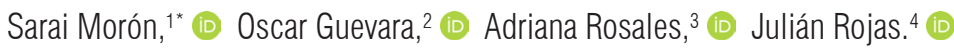

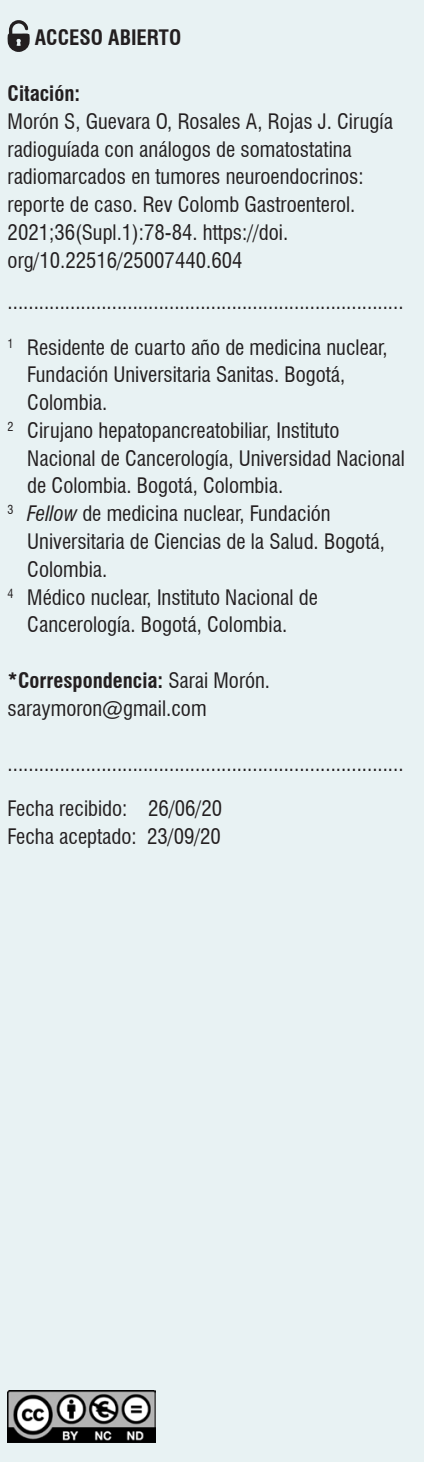

\begin{abstract}
Resumen
Introducción: el mejor tratamiento para los tumores neuroendocrinos es la resección completa del tumor, los ganglios, e inclusive en casos seleccionados, las metástasis a distancia. En ocasiones, el tumor primario es pequeño y de difícil localización preoperatoria o sus recaídas pueden ser difíciles de localizar en el terreno de fibrosis por cirugías o tratamientos previos. La cirugía radioguíada ofrece una opción adicional de localización intraoperatoria que hasta ahora no ha sido muy utilizada en tumores neuroendocrinos. Presentación del caso: paciente de 59 años con antecedente de resección atípica de duodeno y páncreas por tumor neuroendocrino grado 2 del duodeno un año antes. En la tomografía por emisión de positrones/tomografía computarizada (PET/CT) ${ }^{68} \mathrm{Ga}-\mathrm{DOTANOC}$ se encontró un ganglio con sobreexpresión de receptores de somatostatina en el mesenterio, sin otras lesiones a distancia. Por los antecedentes quirúrgicos y la dificultad de visualizar la lesión en las imágenes anatómicas (resonancia magnética [RM]) se decidió realizar la cirugía radioguíada. En el preoperatorio se administraron $15 \mathrm{mCi}$ de tecnecio 99 metaestable-hidrazinonicotinilo-Tyr3-octreotida ( ${ }^{99 m}$ Tc-HYNIC-TOC) y se verificó la buena captación en el ganglio. En cirugía, luego de la disección inicial se utilizó la sonda gamma, que detectó una actividad 5 veces mayor en el ganglio, comparado con los tejidos vecinos, lo que permitió su localización y resección. La evolución fue adecuada y un año después no hay evidencia de recaídas. Conclusión: la cirugía radioguíada no ha sido muy utilizada en la localización intraoperatoria de tumores neuroendocrinos, pero es una buena alternativa en casos seleccionados, como el presentado en este artículo, y permite la detección intraoperatoria y su resección completa.
\end{abstract}

\section{Palabras clave}

Tumores neuroendocrinos, medicina nuclear, cirugía radioguiada, tomografía por emisión de positrones.

\section{Abstract}

Introduction: The best treatment for neuroendocrine tumors is complete resection of the tumor, lymph nodes, and even distant metastases in selected cases. Sometimes, the primary tumor is small and difficult to detect before surgery, or its relapses may be difficult to locate in the fibrosis field due to previous surgeries or treatments. Although radioguided surgery allows for additional intraoperative localization, it has yet to be widely used in neuroendocrine tumors. Case report: A 59-year-old patient with a history of atypical resection of duodenum and pancreas due to grade 2 neuroendocrine tumor of the duodenum one year earlier. On 68Ga-DOTANOC PET/CT, a node with somatostatin receptor overexpression was found in the mesentery, with no other distant lesions. Due to the surgical history and the difficulty in visualizing the lesion on anatomical images (MRI), it was decided to perform the radioguided surgery. During the preoperative period, $15 \mathrm{mCi}$ of 99mTC-HYNIC-TOC were administered verifying good uptake in the ganglion. Following the initial dissection, a gamma probe was used, detecting 5 times more activity in the ganglion than in adjacent tissues, allowing for localization and resection. The patient's progress was satisfactory, and one year later there is no evidence of relapse. Conclusion: Although radioguided surgery is not commonly used in the intraoperative location of neuroendocrine tumors, it is a viable option in some situations, such as the one presented here, because it allows for intraoperative detection and full resection.

\section{Keywords}

Neuroendocrine Tumors; Nuclear Medicine; Radioguided Surgery; Positron Emission Tomography. 


\section{INTRODUCCIÓN}

Los tumores neuroendocrinos (TNE) son un grupo heterogéneo de neoplasias originadas en las células neuroendocrinas. La mayoría de los TNE sobreexpresan receptores de somatostatina, fundamentalmente los tipos 2 y 5 (1). La Sociedad Europea de TNE (ENETS) recomienda la resección curativa del tumor con extirpación del tumor primario, ganglios linfáticos regionales y metástasis hepáticas, cuando sea factible (2).

Sin embargo, las laparotomías recurrentes conducen a múltiples adherencias y alteran la anatomía, por lo que es difícil para los cirujanos diferenciar el tejido cicatricial o inflamatorio del maligno. El uso exitoso de la cirugía radioguíada (CRG) en otros procedimientos quirúrgicos, como la detección de ganglios centinelas, de cáncer de tiroides y de adenoma paratiroideo, ha llevado a proponer el uso de la CRG en los TNE (3).

La intervención quirúrgica en pacientes con TNE gastroenteropancreáticos puede ser un desafío en varios escenarios clínicos. Por una parte, algunos pacientes pueden tener tumores pequeños difíciles de localizar durante la exploración quirúrgica (4). Por otra parte, la localización preoperatoria puede estar basada solamente en las imágenes funcionales, sin ser localizadas en imágenes convencionales (tomografía axial computarizada [TAC], ecografía, resonancia magnética nuclear $[\mathrm{RMN}]$ ). La ubicación de las lesiones puede ser difícil en sitios como la raíz mesentérica y el retroperitoneo (5).

Sin embargo, la introducción de imágenes híbridas preoperatorias (tomografía por emisión de fotón único/ tomografía computarizada [SPECT/CT] o tomografía por emisión de positrones/tomografía computarizada [PET/ $\mathrm{CT}]$ ) ha mejorado aún más la precisión de las técnicas de CRG, que conducen a la resección de tumores primarios pequeños, tumores residuales, recaídas locorregionales y recurrencias distantes (6).

Por tratarse de una técnica innovadora, se describe el caso clínico de un paciente con TNE del Instituto Nacional de Cancerología, Bogotá, Colombia (Figura 1). Para este reporte se siguieron en su mayoría los lineamientos de la guía CARE para reporte de casos (7).

\section{CASO CLÍNICO}

A continuación, se presenta el caso de un paciente de 59 años quien presentó dos episodios de sangrado digestivo, uno en junio de 2015 y otro en febrero de 2017. Se realizó una endoscopia de vías digestivas altas (EVDA) en la que se evidenció en ambos procedimientos una úlcera en la tercera porción del duodeno no susceptible de biopsia. En febrero de 2017 se realizó una TAC de abdomen extra- institucional que evidenció una masa quística que no realza con el contraste, con medidas de $44 \times 32$ mm rodeando parcialmente el cuello del páncreas y otra masa de 26 x $15 \mathrm{~mm}$ con un leve realce en la fase arterial, en la luz de la primera porción del duodeno. En marzo de 2017 el paciente fue llevado a pancreatectomía parcial, duodenectomía parcial, antrectomía y vaciamiento ganglionar en otra institución. El reporte de la patología es de un TNE bien diferenciado de duodeno grado 2 (Organización Mundial de la Salud [OMS], 2017), unifocal de $3 \times 1,2 \mathrm{~cm}$ con compromiso hasta la muscular propia, sin invasión linfovascular ni perineural, con índice mitótico de 1 x 10 cap; bordes de sección negativos, inmunohistoquímica (IHQ): Ki-67 del $10 \%$ y positivo para sinaptofisina y citoqueratina $(\mathrm{CK}) \mathrm{AE} 1 / \mathrm{AE} 3$; negativo para cromogranina.

En septiembre de 2017 presentó deposiciones melénicas, por lo que se realizó una nueva EVDA en la que se encontró mucosa fundocorporal con eritema en parches, remanente del antro gástrico y luz intestinal normal. En octubre de 2017 se realizó una gammagrafía de receptores de somatostatina extrainstitucional, la cual presentó una captación en el mesogastrio paramedial derecho, Krenning 3/4, positivo para sobreexpresión de receptores de somatostatina, sugestiva de recurrencia tumoral. Por consiguiente, se realizó un PET/CT ${ }^{68} \mathrm{Ga}$-DOTANOC (3,5 mCi) (Figura 2) en abril de 2018 y se encontró un ganglio con sobreexpresión de receptores de somatostatina localizado en el mesenterio, por encima de la tercera porción del duodeno con medidas de 20 × 22 × $18 \mathrm{~mm}$ y valor máximo estandarizado de captación $\left(\mathrm{SUV}_{\text {máx }}\right)$ de 57,6 (Krenning 4/4), compatible con persistencia tumoral. No se observaron otras lesiones con sobreexpresión de receptores de somatostatina en el lecho quirúrgico o a distancia.

Debido a que en las imágenes de TAC de abdomen de septiembre de 2017 no se evidenció el ganglio ya que no era fácilmente visible y debido a la incertidumbre de la anatomía posoperatoria o el tipo de reconstrucción digestiva que tenía, se planeó realizar una CRG luego de una discusión de la junta multidisciplinaria de TNE.

El paciente fue llevado a resección de un ganglio mesentérico por laparotomía en agosto de 2018. Se administraron $15 \mathrm{mCi}$ de tecnecio 99 metaestable-hidrazinonicotiniloTyr3-octreotida ( ${ }^{99 \mathrm{~m}} \mathrm{Tc}-\mathrm{HYNIC}-\mathrm{TOC}$ ) intravenoso 5 horas antes de la cirugía (Figura 3) y se localizó la lesión por medio de una sonda gamma. Los hallazgos intraoperatorios fueron un ganglio mesentérico de $2 \mathrm{~cm}$, con una actividad de 3052 cuentas que fue registrada por la sonda gamma. El tejido vecino de la lesión presenta una actividad menor de 700 cuentas (Figura 4). La patología confirmó un ganglio linfático comprometido por TNE, conteo mitótico: 2 mitosis en 10 cap; índice de proliferación celular (Ki-67): $10 \%$, grado 2 de la OMS, 2017. 


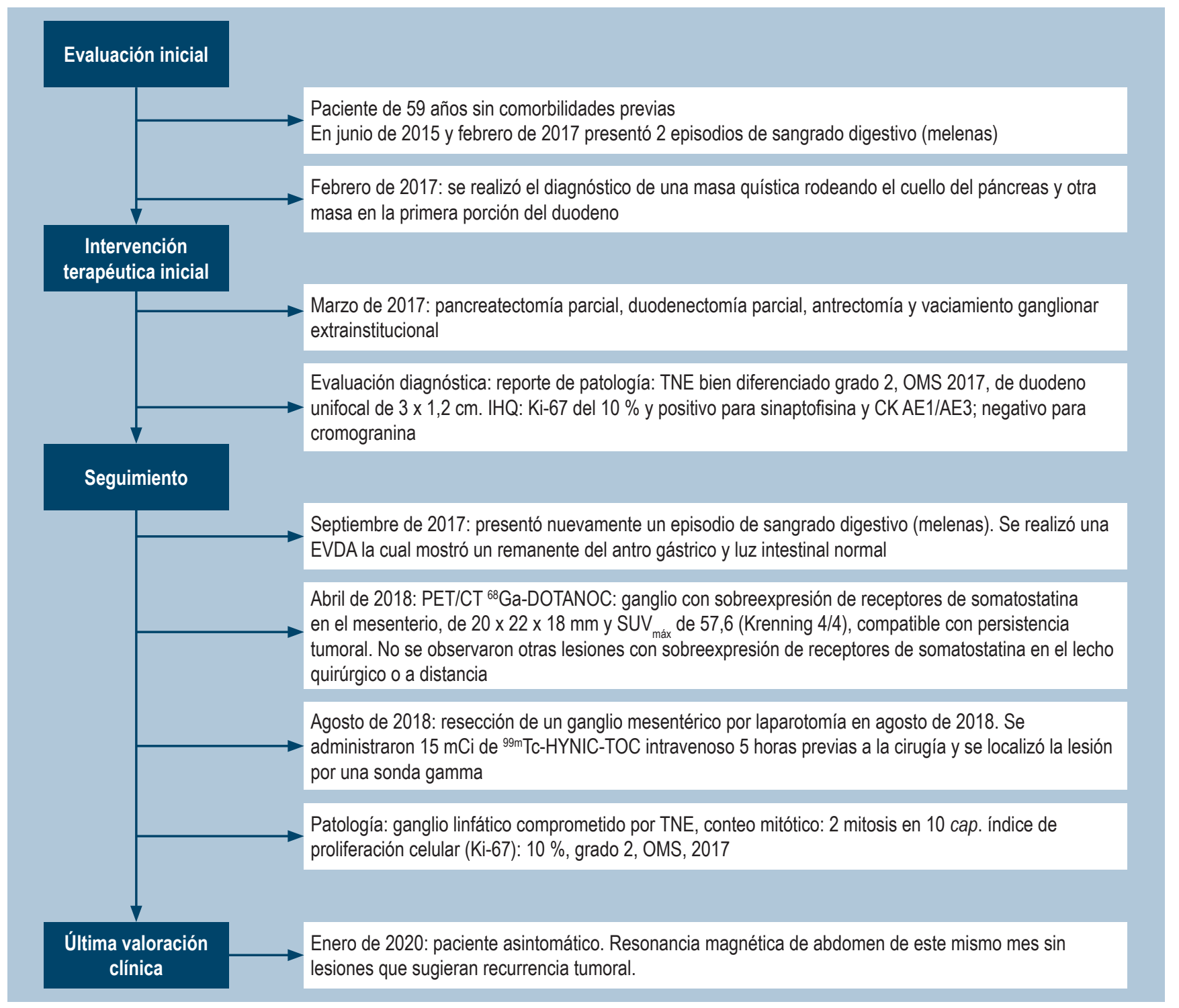

Figura 1. Flujograma de la evolución clínica del paciente.

Después de la CRG, el paciente continuó en seguimiento por el servicio de endocrinología oncológica y gastroenterología de la institución. La última valoración fue en enero de 2020 con RMN de abdomen del mismo mes que no evidenció lesiones que sugirieran una recurrencia tumoral. Actualmente, el paciente se encuentra asintomático.

\section{DISCUSIÓN}

La incidencia de TNE gastroenteropancreáticos ha aumentado aproximadamente a 7,8 casos por 100000 personas cada año y la prevalencia es de aproximadamente 35 casos por cada 100000 personas (8).
La resección quirúrgica es la mejor opción de tratamiento curativo para pacientes con TNE en etapa temprana. La extirpación completa del tumor es un factor pronóstico importante en pacientes con TNE gastroenteropancreáti$\cos (9)$, ya que mejora la calidad de vida y reduce la incidencia de metástasis. Por esta razón, la capacidad de lograr la resección $\mathrm{R} 0$ o $\mathrm{R} 1$ se ha relacionado con mejores resultados en la supervivencia (3).

Asimismo, la determinación de la extensión del tumor (localización y metástasis) y la ubicación del tumor primario es esencial en el manejo de los TNE. Ciertas localizaciones, como el intestino delgado, pueden asociarse con multicentricidad y se debe tener cuidado para garantizar 


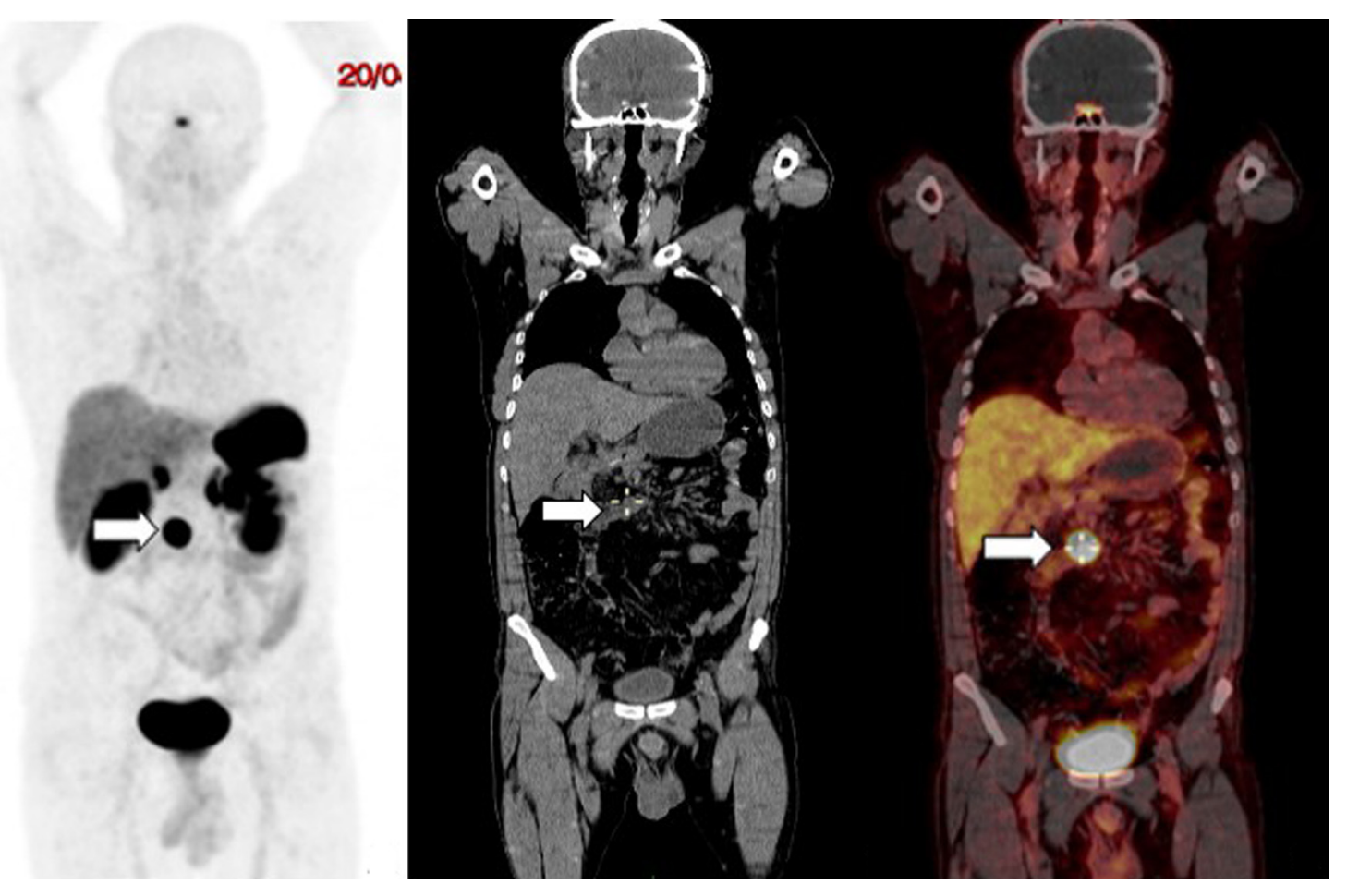

Figura 2. Paciente de 59 años, con TNE de duodeno. La PET/CT con ${ }^{68} \mathrm{Ga}$-DOTANOC mostró un ganglio con sobreexpresión de receptores de somatostatina, localizado en el mesenterio (flechas), por encima de la tercera porción del duodeno de 20 x $22 \mathrm{x}$ $18 \mathrm{~mm} \mathrm{SUV}_{\text {max }}$ de 57,6 (Krenning 4/4). A. Imagen de máxima intensidad. B. Corte coronal de TAC de baja dosis. C. Imagen de fusión de PET/CT.

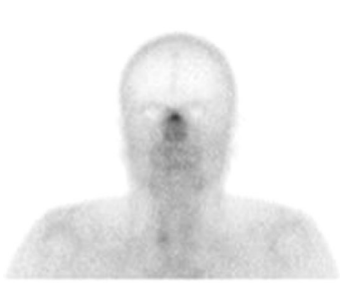

CYC ANT

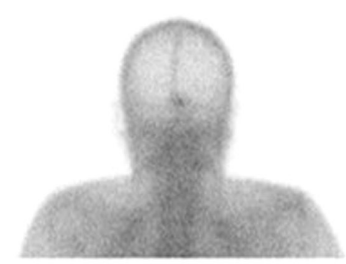

CYCPOST:

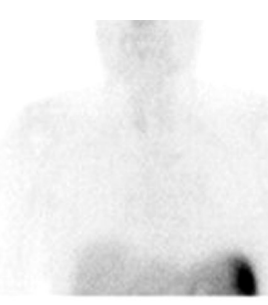

TORAXANT

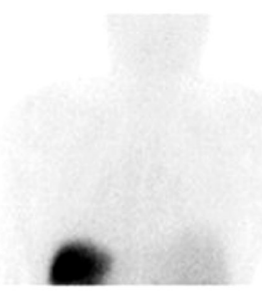

TORAXPOST

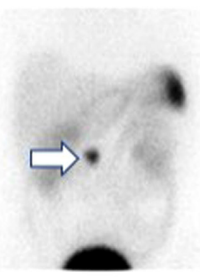

ABDOMENANT

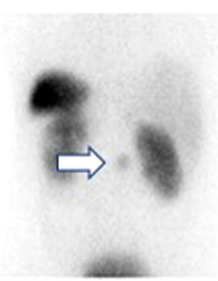

ABDOMEN POST

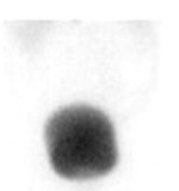

PELVS ANT

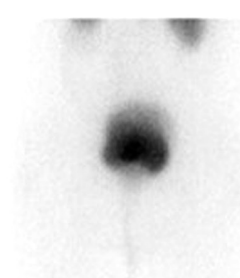

PELVIS POST

Figura 3. Gammagrafía de receptores de somatostatina con ${ }^{99 m}$ Tc-HYNIC-TOC el día de la cirugía. Se muestra la captación en el mesogastrio (flechas) correspondiente a un ganglio con sobreexpresión de receptores de somatostatina (Krenning 4/4) ya conocido en la PET/CT con ${ }^{68} \mathrm{Ga}$-DOTANOC previo. 


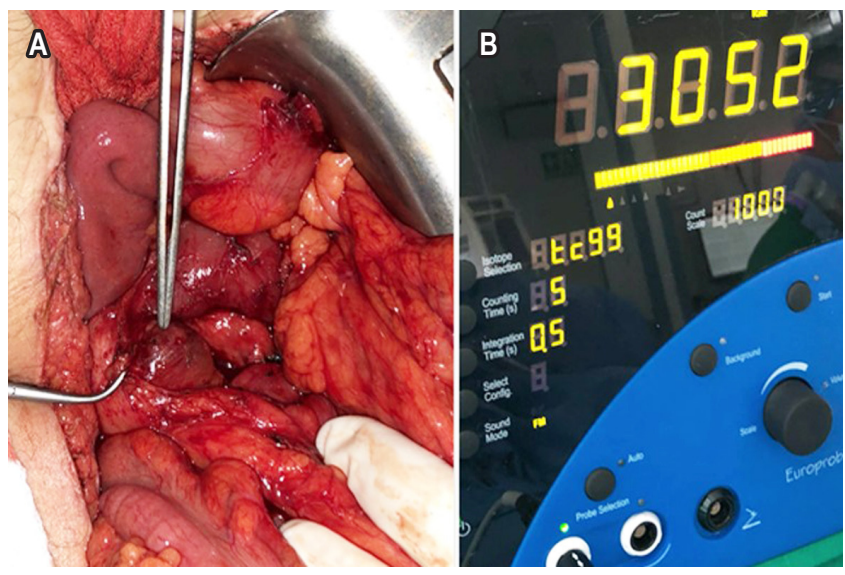

Figura 4. A. Radiolocalización exitosa de adenopatía mesentérica. B. Sonda gamma utilizada.

una adecuada resección (10). La localización intraoperatoria puede lograrse usando técnicas de cirugía tradicional, radiológicas y endoscópicas, que incluyen palpación, marcación endoscópica y ultrasonido intraoperatorio (11).

Además de lo anterior, los TNE se pueden detectar con técnicas de CRG mediante el uso de radiotrazadores y una sonda de detección gamma (11). El radiofármaco se administra antes de la cirugía o intraoperatoriamente, y la sonda gamma se usa para detectar el tumor primario, la afectación ganglionar o la enfermedad metastásica (12).

La CRG ha demostrado su utilidad en TNE, tanto en la detección de tumores ocultos como de pequeño tamaño (1). Esta es una técnica para optimizar la identificación y resección quirúrgica completa de todos los posibles sitios de la enfermedad (13). En el contexto de la recaída tumoral o ganglionar, se realiza previamente a la CRG una estadificación con imágenes funcionales con PET/CT ${ }^{68} \mathrm{Ga}$ dotapéptidos en todos los pacientes, el cual valora la expresión de receptores de somatostatina y la extensión de la enfermedad, si está localizada o si presenta enfermedad a distancia. En el caso de que la enfermedad esté localizada en un sitio de difícil acceso o la lesión sea de pequeño tamaño, se considera que es el paciente ideal para realizar la resección con CRG. En este paciente en quien ya se había realizado la resección del tumor primario y con exámenes de gammagrafía ${ }^{99} \mathrm{Tc}-\mathrm{HYNIC}$-TOC y PET/CT ${ }^{68} \mathrm{Ga}$ dota-péptido previos que no mostraban compromiso de otra lesión, se realizó la resección del ganglio comprometido, teniendo en cuenta su localización en la raíz del mesenterio, lo que hace difícil realizar alguna linfadenectomía formal. Están indicadas las linfadenectomías de los ganglios periduodenales durante la resección del tumor primario.

La CRG es la aplicación de la medicina nuclear que, junto con la PET/CT, ha tenido un crecimiento relevante en los últimos 25 años. El término $C R G$ incluye un conjunto de técnicas pre-, intra- y posoperatorias, cuya característica principal es la inyección de un radiofármaco asociado con el uso intraoperatorio de una sonda portátil de conteo de radiactividad (conocido como sonda gamma) que permite que los cirujanos identifiquen y remuevan los tejidos diana que acumulan la radiactividad (6). La sonda gamma proporciona información visual y audible de la tasa de recuento de radiactividad (rango 0-25 000 cuentas por segundo [cps]) a medida que el tumor es abordado por el cirujano (9).

La ubicación de la lesión con la sonda gamma intraoperatoria requiere una relación entre las cps del tumor/cps del fondo de al menos 1,5 para confirmar dicha localización (5). En nuestro caso en mención, la relación tumor/fondo fue de 4,3, por lo que la localización del ganglio fue exitosa.

La exploración con la sonda gamma ha identificado un 57 $\%$ más lesiones de TNE en comparación con la exploración manual por el cirujano; asimismo, podría identificar aquellas lesiones que miden entre 0,5 y $1 \mathrm{~cm}$ con alta eficiencia (5). Por consiguiente, el uso de las sondas gamma tiene un alto grado de especificidad y se puede utilizar para ayudar a los cirujanos a localizar pequeñas lesiones tumorales, que son difíciles de ubicar a simple vista (9).

En uno de los estudios más grandes, con 44 pacientes $(22$ de origen gastrointestinal) utilizando galio 68-DOTATATE, se encontró que con una relación tumor/tejido vecino de 2,5, la sensibilidad fue del $90 \%$ y la especificidad fue del 25 $\%$; y con una relación de 16, la sensibilidad descendió al 54 $\%$, pero la especificidad aumentó al $81 \%$ (4).

La CRG con análogos de somatostatina radiomarcados está disponible con diferentes radiotrazadores como ${ }^{111}$ In-pentetreotida, ${ }^{99 \mathrm{~m}} \mathrm{Tc}$-análogos de somatostatina y ${ }^{68} \mathrm{Ga}$-análogos de somatostatina (13); este último con mayor tasa de detección porque tiene mayor energía de emisión. La tasa de detección es del 94 \% (3), la cual es mayor en comparación con los otros radiotrazadores. Sin embargo, en nuestro medio aún no se encuentra disponible.

En el caso mencionado, el radiofármaco que se usó fue ${ }^{99 m}$ Tc-HYNICTOC, que es un análogo de la somatostatina. Este fue desarrollado inicialmente por Behé y Maecke (14) en el 2000, el cual posee adecuadas características clínicas como elevada y específica afinidad por receptores de somatostatina, buena biodistribución, excreción renal, baja exposición a la radiación, disponibilidad y costo-efectividad. Este trazador proporciona, además de alta calidad de imagen, un diagnóstico más temprano (imágenes a los 10 minutos-4 horas) (1). El uso de este radiofármaco se ha reportado poco en la literatura, con 9 casos en el intestino delgado (1), por lo cual vale la pena destacar su utilidad.

En nuestro paciente se pudo realizar la resección de una adenomegalia mesentérica exitosa gracias a la CRG con 
análogos de receptores de somatostatina radiomarcados. Sin embargo, existen otros factores para la realización de una resección exitosa, dentro de los que se encuentran el apropiado diagnóstico preoperatorio para localizar los sitios de la enfermedad, la obtención adecuada de exposición de la lesión durante la cirugía y el rendimiento de la sonda gamma para la detección de las lesiones (13).

En caso de que no sea posible la CRG porque no está disponible, los cirujanos usan la palpación manual o el ultrasonido intraoperatorio para identificar pequeñas lesiones; sin embargo, la detección correcta puede ser un reto aun en casos en los que se cuenta con cirujanos expertos, debido al tamaño milimétrico y múltiples localizaciones en el mismo paciente. Aunque faltan muchos estudios, es necesario un análisis más profundo para aclarar el rendimiento de la CRG, si esta aumenta el número de resecciones y en qué afecta el procedimiento quirúrgico, así como la supervivencia de dichos pacientes (15).

En el futuro, estudios prospectivos con cohortes más grandes de pacientes plantearán la eficacia de la CRG para minimizar los síntomas, el efecto sobre la calidad de vida y la supervivencia global junto con el riesgo intra- o perioperatorio (3). A largo plazo, se requiere el seguimiento y la comparación con pacientes similares que no tuvieron CRG para determinar si este enfoque terapéutico reduce la tasa de enfermedad persistente o recurrente en pacientes con TNE (4). Dada la baja frecuencia de estas presentaciones, se considera poco factible la opción de realizar un ensayo clínico aleatorizado.

En estas patologías es importante la discusión multidisciplinaria de los casos para buscar las mejores opciones diagnósticas y terapéuticas, así como también debe garantizarse este tipo de tratamientos en centros con suficiente experticia y recursos, como en nuestra institución. En este caso, fue importante esta interacción entre las diferentes especialidades para obtener la resección completa de la lesión.

\section{CONCLUSIÓN}

La CRG es una técnica factible en la localización de TNE, que detecta más lesiones y de menor tamaño que las pruebas de imagen prequirúrgicas y la palpación por el cirujano. El ${ }^{99 \mathrm{~m}} \mathrm{Tc}-\mathrm{HYNICTOC}$ es un radiofármaco útil en la localización intraoperatoria de TNE intestinales, hasta ahora poco reportado en la literatura, pero que se convierte en una alternativa en la localización de dichos tumores.

\section{REFERENCIAS}

1. García-Talavera P, Ruano R, Rioja ME, Cordero JM, Razola $\mathrm{P}$, Vidal-Sicart S. Cirugía radioguíada de tumores neuroendocrinos. Revisión de la literatura. Rev Esp Med Nucl Imagen Mol. 2014;33(6):358-65.

https://doi.org/10.1016/j.remn.2014.07.004

2. Niederle B, Pape UF, Costa F, Gross D, Kelestimur F, Knigge U, Öberg K, Pavel M, Perren A, Toumpanakis C, O'Connor J, O'Toole D, Krenning E, Reed N, Kianmanesh R; Vienna Consensus Conference participants. ENETS Consensus Guidelines Update for Neuroendocrine Neoplasms of the Jejunum and Ileum. Neuroendocrinology. 2016;103(2):125-38. https://doi.org/10.1159/000443170

3. Kaemmerer D, Prasad V, Daffner W, Haugvik SP, Senftleben $S$, Baum RP, Hommann M. Radioguided surgery in neuroendocrine tumors using Ga-68-labeled somatostatin analogs: a pilot study. Clin Nucl Med. 2012;37(2):142-7. https://doi.org/10.1097/RLU.0b013e3182291de8

4. El Lakis M, Gianakou A, Nockel P, Wiseman D, Tirosh A, Quezado MA, Patel D, Nilubol N, Pacak K, Sadowski SM, Kebebew E. Radioguided Surgery With Gallium 68 Dotatate for Patients With Neuroendocrine Tumors. JAMA Surg. 2019; 154(1):40-45.

https://doi.org/10.1001/jamasurg.2018.3475
5. Gulec SA, Baum R. Radio-guided surgery in neuroendocrine tumors. J Surg Oncol. 2007;96(4):309-15. https://doi.org/10.1002/jso.20868

6. Valdés Olmos RA, Vidal-Sicart S, Manca G, Mariani G, León-Ramírez LF, Rubello D, Giammarile F. Advances in radioguided surgery in oncology. QJ Nucl Med Mol Imaging. 2017;61(3):247-70. https://doi.org/10.23736/S1824-4785.17.02995-8

7. Gagnier JJ, Kienle G, Altman DG, Moher D, Sox H, Riley D; CARE Group*. The CARE Guidelines: Consensusbased Clinical Case Reporting Guideline Development. Glob Adv Health Med. 2013;2(5):38-43. https://doi.org/10.7453/gahmj.2013.008

8. Tsikitis VL, Wertheim BC, Guerrero MA. Trends of incidence and survival of gastrointestinal neuroendocrine tumors in the United States: a seer analysis. J Cancer. 2012;3:292-302. https://doi.org/10.7150/jca.4502

9. Sadowski SM, Millo C, Neychev V, Aufforth R, Keutgen X, Glanville J, Alimchandani M, Nilubol N, Herscovitch P, Quezado M, Kebebew E. Feasibility of Radio-Guided Surgery with ${ }^{68}$ Gallium-DOTATATE in Patients with Gastro-Entero-Pancreatic Neuroendocrine Tumors. Ann Surg Oncol. 2015;22 Suppl 3(Suppl 3):S676-82. https://doi.org/10.1245/s10434-015-4857-9

10. Makridis C, Oberg K, Juhlin C, Rastad J, Johansson H, Lörelius LE, Akerström G. Surgical treatment of mid-gut 
carcinoid tumors. World J Surg. 1990;14(3):377-83; discussion 384-5.

https://doi.org/10.1007/BF01658532

11. Adams S, Baum RP, Hertel A, Wenisch HJ, Staib-Sebler E, Herrmann G, Encke A, Hör G. Intraoperative gamma probe detection of neuroendocrine tumors. J Nucl Med. 1998;39(7):1155-60.

12. Baum RP, Sandrucci S, Adams S. Radioguided Surgery of Neuroendocrine Tumors. En: Mariani G, Giuliano AE, Strauss HW (editores). Radioguided Surgery: A Comprehensive Team Approach. 1. ${ }^{\text {a }}$ edición. Nueva York: Springer; 2008. p. 252-61. https://doi.org/10.1007/978-0-387-38327-9_24

13. Hall NC, Bluemel C, Vidal-Sicart S, Povoski SP. Radioguided Surgery for Gastroenteropancreatic Neuroendocrine Tumors. En: Herrmann K, Nieweg O,
Povoski SP (editores). Radioguided Surgery: Current Applications and Innovative Directions in Clinical Practice. 1. a edición. Nueva York: Springer; 2016. p. 299-311. https://doi.org/10.1007/978-3-319-26051-8_19

14. Bangard M, Béhé M, Guhlke S, Otte R, Bender H, Maecke HR, Biersack HJ. Detection of somatostatin receptorpositive tumours using the new $99 \mathrm{mTc}$-tricine-HYNIC-DPhe1-Tyr3-octreotide: first results in patients and comparison with 111 In-DTPA-D-Phe1-octreotide. Eur J Nucl Med. 2000;27(6):628-37. https://doi.org/10.1007/s002590050556

15. Ambrosini V, Fanti S. Radioguided surgery with 68Ga-DOTATATE for patients with neuroendocrine tumors. Hepatobiliary Surg Nutr. 2020;9(1):67-69. https://doi.org/10.21037/hbsn.2019.06.04 